\title{
The Diffuse Scattering in Electron Diffraction from the High-Temperature Phase of Silver Selenide
}

\author{
By R. DE Ridder and S. Amelinckx \\ Rijksuniversitair Centrum (R.U.C.A.), Middelheimlaan 1, B-2020 Antwerpen, Belgium
}

(Received 29 April 1974)

The diffuse scattering as observed in electron diffraction patterns of $\alpha-\mathrm{Ag}_{2} \mathrm{Se}$ is associated with a disordered silver-vacancy system. Because of the disorder the silver atoms are displaced from their crystallographic positions and one has to account for: (a) a characteristic diffuse scattering which can be treated in terms of the kinematical Warren-Averbach-Roberts theory (size displacement effect) [Warren, B. E., Averbach, B. L. \& Roberts, B. W. (1951). J. Appl. Phys. 22, 1493; Borie, B. (1957). Acta Cryst. 10, 89; Borie, B. \& Sparks C. J. Jr (1971). Acta Cryst. A 27, 198]; (b) a change in the Bragg intensities from the presence of Huang scattering in the neighbourhood of the Bragg reflections [Rahlfs, P. (1936). Z. Phys. Chem. 31B, 157]. In relation to the general features and details of the diffuse intensity distribution the structural model for $\alpha-\mathrm{Ag}_{2} \mathrm{Se}$ as proposed by Rahlfs is reviewed.

J. Appl. Cryst. (1975). 8, 211

\section{Lattice Relaxation at Antiphase Boundaries in Ordered Ni-Mo Alloys}

\author{
By G. van Tendeloo and S. Amelinckx \\ Rijksuniversitair Centrum (R.U.C.A.), Middelheimlaan 1, B-2020 Antwerpen, Belgium
}

(Received 29 April 1974)

\begin{abstract}
Different types of antiphase boundaries have been studied by electron microscopy in the alloys $\mathrm{Ni}_{3} \mathrm{Mo}$ and $\mathrm{Ni}_{4} \mathrm{Mo}$ [Van Tendeloo, G., Delavignette, P., Gevers, R., \& Amelinckx, S. (1973). Phys. Stat. Sol. (a), 18, 85; Ruedl, E., Delavignette, P. \& Amelinckx, S. (1968). Phys. Stat. Sol. 28, 305]. Thermal boundaries as well as glide boundaries exhibit a residual contrast when two-beam diffraction conditions are used for a fundamental reflexion [Van Tendeloo, G. \& Amelinckx, S. (1974). Phys. Stat. Sol. In the press]. From these observations it could be deduced that some relaxation of the lattice has occurred. The presence of excess molybdenum along the interface gives rise to a small supplementary displacement vector $\bar{\varepsilon}$, the total displacement vector for the fault then being $\mathbf{R}+\bar{\varepsilon}$. The direction and the sense of $\bar{\varepsilon}$ have been determined from diffraction experiments; for all different situations the supplementary displacement is equivalent to a small expansion of the boundary regions. Glide antiphase boundaries having a particular orientation seem to have a larger $\bar{\varepsilon}$ vector than the thermal boundaries, which is shown to be consistent with the structural model of the defects. For the $\mathrm{Ni}_{4} \mathrm{Mo}$ alloy, where the thermal interfaces are not so strictly confined to crystallographic planes, the dependence of $\bar{\varepsilon}$ on the orientation of the fault is striking.
\end{abstract}

